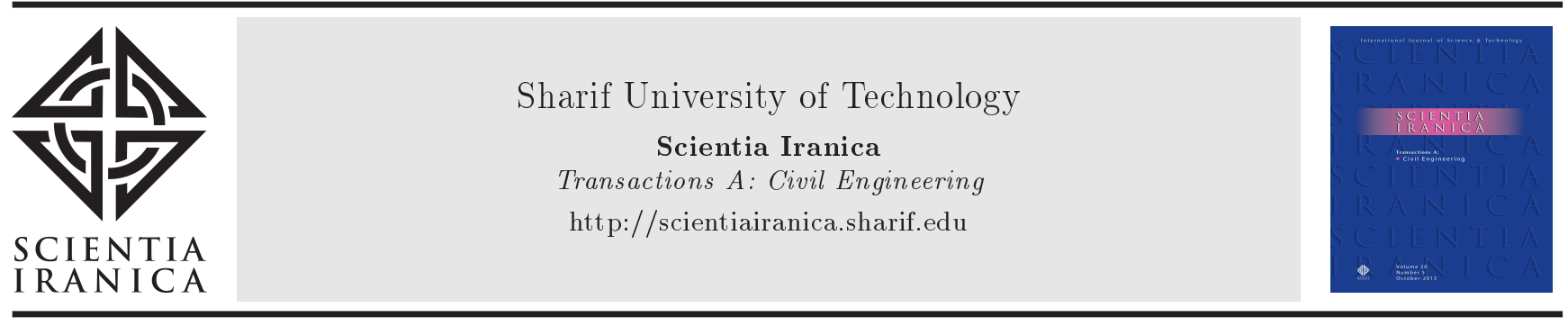

\title{
Application of smoothed finite element method in coupled hydro-mechanical analyses
}

\author{
E. Karimian and M. Oliaei* \\ Department of Civil and Environmental Engineering, Tarbiat Modares University, Tehran, P.O. Box 14115-116, Iran.
}

Received 13 February 2017; received in revised form 8 June 2017; accepted 15 July 2017

KEYWORDS
Smoothed finite
element method;
The finite element
method;
Coupled hydro-
mechanical analysis;
Consolidation;
Biot's theory.

\section{Introduction}

The Finite Element Method (FEM) has been widely used to solve partial differential equations for many years. Although this method has been applied widely, its mesh dependency has restricted its application in some problems. For example, when there are large deformations or crack propagation, the method cannot be used effectively. To overcome these shortcomings, different types of Mesh-free Methods (MMs) have been developed (e.g., [1-4]).

\footnotetext{
*. Corresponding author. Tel.: +982182884395; Fax: +982182884395

E-mail addresses: e.karimian@modares.ac.ir (E. Karimian); m.olyaei@modares.ac.ir (M. Oliaei)
}

In some types of MMs, computing shape functions and a large number of Gauss points that are required to decrease the integration error of the discretized weak form increase their computational cost, compared to the FEM $[1,2]$.

To eliminate the Gauss integration, nodal integration of the EFG method was proposed by Beissel and Belytschko [5], which eliminates integration on background cells. Direct nodal integration often causes numerical instability, because the derivatives of the shape functions vanish at the nodes. It also decreases convergence rate and accuracy due to the violation of the integration constraints in the Galerkin weakform formulations. To avoid these shortcomings, Chen et al. [6] introduced Stabilized Conforming Nodal Integration (SCNI). The SCNI uses a strain measure calculated as the spatial average of the compatible strain field (the symmetric gradient of the 
displacements). This technic is called "Strain Smoothing".

Liu et al. [7], using incompatible Point Interpolation Method (PIM) shape functions and the SCNI, introduced a mesh-free linearly conforming point interpolation method, which was later called the Smoothed Point Interpolation Method (SPIM) [8]. Later, by application of the SCNI (which was successfully applied in MMs) in the FEM, the Smoothed Finite Element Method (SFEM) was introduced [9].

Based on how the strain smoothing procedure is done, there are three main types of SFEM:

1. Cell based SFEM (CSFEM) [9];

2. Node based SFEM (NSFEM) [10];

3. Edge based SFEM (ESFEM) [11].

In the CSFEM, strain smoothing is done on domains that are created by dividing the elements of the original FEM mesh into sub-cells; the NSFEM uses nodes to create smoothing domains; and in the ESFEM, smoothing domain creation is based on the edges of the elements.

SFEMs have some interesting properties. They are insensitive to mesh distortion, because isoparametric mapping used in the conventional FEM is not needed. Using the divergence theorem, area integration on smoothing domains reduces to integration on boundaries, and shape function derivatives are not required. SFEMs are generally more computationally efficient than MMs and even the FEM, for the same accuracy level [8].

Because of their interesting features, SFEMs have been applied to solve different problems. Problems such as vibration and dynamic analysis [12,13], elasticplastic analysis [14,15], fracture mechanics [16-18], heat transfer [19,20], structural acoustics [21-23], contact problems [24], adaptive analysis $[25,26]$, impact problem [27], and many more. These researches show that, compared with the FEM, SFEMs have many advantages in treating different problems, specifically when there are large deformations and nonlinear material behavior. On the other hand, like any other numerical method, SFEMs may have some drawbacks. Some of them, which are related to the solution to coupled problems, are discussed later in this paper. Investigating other possible shortcomings, for example excessive damping of sharp gradients, needs further research.

In this paper, using a developed SFEM/FEM code, different types of smoothed finite element method are applied to solve the consolidation problem. Performance of different strategies for application of these methods in the solution to coupled hydro-mechanical problems is studied through the solution to a number of examples. Results are compared with those obtained using the FEM and analytical methods and the best scenario for incorporation of SFEMs in consolidation analyses is indicated.

Following the introduction, Biot's consolidation theory as well as its formulation and equations is reviewed. Theories and formulations of the smoothed finite element method are presented next. Later, the discretized form of the coupled hydro-mechanical equations of consolidation, using SFEM for spatial discretization, is obtained. In the last section, some examples are solved and results are investigated. Finally, conclusion is given.

\section{Biot's consolidation theory}

Biot's theory of consolidation [28] explains the coupled hydro-mechanical behavior of elastic saturated porous media. It combines equation of equilibrium, Terzaghi's principle of effective stress, fluid continuity equation, and Darcy's seepage law to develop two equations with displacement and pore water pressure as the main variables.

The first governing equation in the Biot's consolidation theory is the equation of equilibrium, which in tensor notation is written as:

$$
\sigma_{i j, j}+b_{i}=0
$$

In this equation, $\sigma_{i j}$ is total stress tensor and $b_{i}$ the unit body force. Terzaghi's principle of effective stress shows the relationship between total stress and pore water pressure in saturated porous media:

$$
\sigma_{i j}=\sigma_{i j}^{\prime}+P \delta_{i j}
$$

where $\sigma_{i j}^{\prime}$ is effective stress tensor, $P$ is pore water pressure, and $\delta_{i j}$ is Kronecker delta. The constitutive equation of solid skeleton is used to obtain displacements, using the equilibrium equation. First, the constitutive equation gives a relationship between stress and strain tensors:

$$
\sigma_{i j}^{\prime}=D_{i j k l} \varepsilon_{k l},
$$

where $\varepsilon_{i j}$ is the strain tensor and $D_{i j k l}$ is the tensor of elastic moduli, because only linear isotropic elastic problems are considered. Then, the relationship between strain and displacements, with the assumption of small displacements and ignoring geometric nonlinearity, is written as:

$$
\varepsilon_{i j}=\frac{1}{2}\left(\frac{\partial u_{i}}{\partial x_{j}}+\frac{\partial u_{j}}{\partial x_{i}}\right) .
$$

Here, $u$ is displacements in $x$ and $y$ directions.

The second governing equation in the Biot's consolidation theory is the fluid continuity equation. It is expressed as:

$$
\dot{\varepsilon}_{v}=q_{i, i}
$$

where $\dot{\varepsilon}_{v}$ is the time derivative of volumetric strain that is written as: 


$$
\varepsilon_{v}=u_{i, i}
$$

and $q_{i}$ is water discharge in the $i$ th direction. Darcy's seepage law gives the relationship between discharge and pore water pressure:

$$
q_{i}=\frac{K_{i j}}{\gamma_{w}} P_{, j}
$$

In this equation, $K_{i j}$ is the permeability tensor of solid skeleton and $\gamma_{w}$ is the unit weight of water.

By combining Eqs. (5)-(7) and writing displacements in incremental form to be integrated over time, the first partial differential equation for coupled hydromechanical analysis of porous media is given as:

$$
\Delta u_{i, i}=\int\left(\frac{K_{i j}}{\gamma_{w}} P_{, j}\right)_{, i} d t
$$

The second governing equation is written by combining equation of equilibrium and Terzaghi's effective stress equation. Since displacements in Eq. (8) appear in incremental form, stresses will be written in incremental form as well:

$$
\sigma_{i j, j}^{\prime t}+\Delta \sigma_{i j, j}^{\prime}+P_{, j} \delta_{i j}+b_{i}=0,
$$

where $\sigma^{t}$ is the effective stress at time step $t$ and $\Delta \sigma^{\prime}$ is the increment of the effective stress.

\section{Smoothed finite element method}

In this section, first, formulation of the finite element method is reviewed. For smoothed finite element, formulation is largely the same and is discussed next. Different types of SFEM are also introduced here.

In a domain of interest $\Omega$ subjected to body forces $b, D$ as a tensor of material moduli, and $\bar{t}$, the known traction on the natural boundary $\Gamma_{t}$, integration is performed over elements to form the discrete equations of the FEM using the Galerkin weak form:

$$
\int_{\Omega}\left(\nabla_{s} \delta u\right)^{T} D\left(\nabla_{s} u\right) d \Omega-\int_{\Omega} \delta u^{T} b d \Omega-\int_{\Gamma_{t}} \delta u^{T} \bar{t} d \Gamma=0 .
$$

Here, $\nabla_{s} u$ is the symmetric gradient of the displacement field, $u$ is a trial function, and $\delta u$ is a test function.

The FEM uses the following trial and test functions:

$$
\begin{gathered}
u^{h}(x)=\sum_{I=1}^{N P} N_{I}(x) d_{I}, \\
\delta u^{h}(x)=\sum_{I=1}^{N P} N_{I}(x) \delta d_{I},
\end{gathered}
$$

where $N P$ is the number of the nodal variables of the element, $d_{I}$ is the nodal displacement vector, and $N_{I}(x)$ is the matrix of shape functions.
By replacing the approximations $u^{h}$ and $\delta u^{h}$ into the weak form, since virtual nodal displacements are arbitrary, the discretized system of equations is obtained:

$$
K^{F E M} d=f,
$$

where $K^{F E M}$ is the element stiffness matrix and $f$ is the element force vector, which are written as:

$$
\begin{aligned}
& K_{I J}^{F E M}=\int_{\Omega} B_{I}^{T}(x) D B_{J}(x) d \Omega, \\
& f_{I}=\int_{\Omega} N_{I}^{T}(x) b d \Omega+\int_{\Gamma_{t}} N_{I}^{T}(x) \bar{t} d \Gamma,
\end{aligned}
$$

the strain matrix $B_{I}(x)$ is defined as:

$$
B_{I}(x)=\nabla_{s} N_{I}(x) .
$$

In SFEM, the integration required in the weak form of the FEM is not performed based on the elements, but is done on the smoothing domains by the strain smoothing technic. In other words, these methods do not use the compatible strains (Eq. (16)), but strains "smoothed" over smoothing domains. Therefore, the stiffness matrix integration is not based on the elements, but it is done on local smoothing domains.

In the cell based SFEM, smoothing domains are created by further dividing the elements of initial mesh into smoothing domains (Figure 1). A major difference between SFEM and the FEM is that in all types of SFEM, elements can be polygons of arbitrary number of sides. The domain $\Omega$ should be discretized into $N_{s}$ smoothing domains in such a way that:

$$
\Omega=\Omega^{(1)} \cup \Omega^{(2)} \cup \cdots \Omega^{\left(N_{s}\right)},
$$

and:

$$
\Omega^{(i)} \cap \Omega^{(j)}=\phi, \quad i \neq j .
$$

It is also required that the number of smoothing domains be greater than the number of elements.

In the node based discretization, after discretization into elements, the domain is divided into smoothing cells associated with nodes, in a non-overlapping

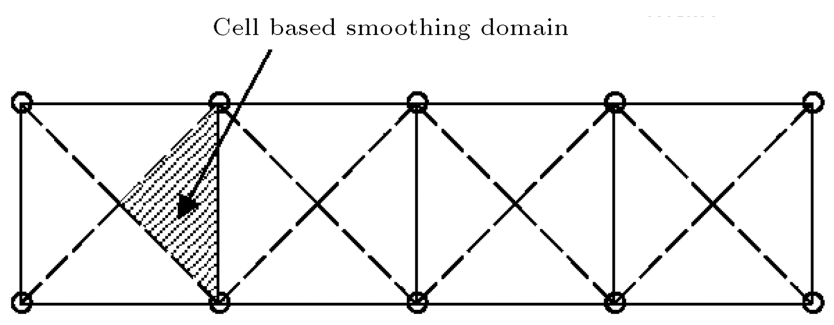

Figure 1. A domain discretized into 10 nodes, 4 elements, and 16 cell based smoothing domains (solid line: element borders, dashed line: smoothing domain borders). 


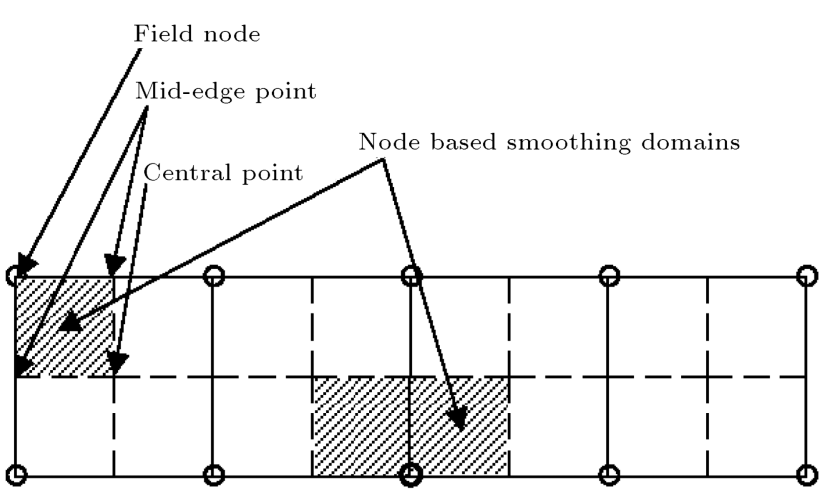

Figure 2. A domain discretized into 10 nodes, 4 elements, and 10 node based smoothing domains (solid line: element borders, dashed line: smoothing domain borders).

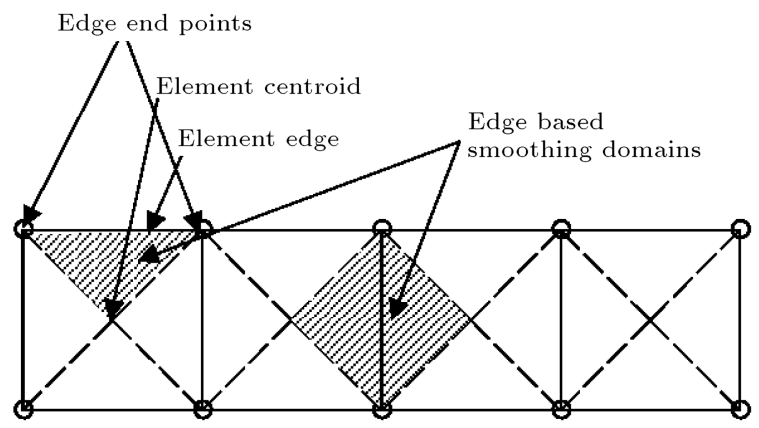

Figure 3. A domain discretized into 10 nodes, 4 elements, and 13 edge based smoothing domains (solid line: element borders, dashed line: smoothing domain borders).

and no-gap manner (as in the CSFEM). For $n$-sided polygonal elements, the cell $\Omega^{(k)}$ associated with the node $k$ is created by connecting sequentially the midedge-point to the central points of the surrounding $n$ sided polygonal elements of the node $k$ as shown in Figure 2. As a result, each $n$-sided polygonal element will be divided into $n$ four-sided sub-domains and each sub-domain is attached to the nearest field node. The cell associated with the node $k$ is then created by combination of each nearest sub-domain of all elements around the node $k$ to each other.

Similarly, in the edge based discretization, the local smoothing domains that should be non-overlapping with no gap between them are constructed based on edges of the elements. For triangular and quadrilateral elements, the smoothing domain $\Omega^{(k)}$ associated with the edge $k$ is created by connecting two end points of the edge to two centroids of two adjacent elements as shown in Figure 3, for quadrilateral elements. Extending the smoothing domain $\Omega^{(k)}$ associated with the edge $k$ to $n$-sided polygonal elements is straightforward.

After creation of the smoothing domains, using the compatible strains and considering $\varepsilon=\nabla_{s} u$, smoothed strains can be obtained by performing the smoothing operation over domain $\Omega^{(k)}$ as:

$$
\tilde{\varepsilon}_{k}=\int_{\Omega^{(k)}} \varepsilon(x) \Phi_{k}(x) d \Omega=\int_{\Omega^{(k)}} \nabla_{s} u(x) \Phi_{k}(x) d \Omega,
$$

where $\Phi_{k}(x)$ is a given smoothing function that satisfies at least unity property:

$$
\int_{\Omega^{(k)}} \Phi_{k}(x) d \Omega=1 .
$$

The simplest local constant smoothing function usually used is [8]:

$$
\Phi_{k}(x)= \begin{cases}\frac{1}{A^{(k)}} & x \in \Omega^{(k)} \\ 0 & x \notin \Omega^{(k)}\end{cases}
$$

Here, $A^{(k)}=\int_{\Omega^{(k)}} d \Omega$ is the area of the smoothing domain $\Omega^{(k)}$. Considering the divergence theorem, by using this function, the smoothed strain can be obtained, which is constant over the domain $\Omega^{(k)}$ :

$$
\tilde{\varepsilon}_{k}=\frac{1}{A^{(k)}} \int_{\Gamma^{(k)}} n^{(k)}(x) u(x) d \Gamma
$$

where $\Gamma^{(k)}$ is the boundary of the domain $\Omega^{(k)}$ and $n^{(k)}(x)$ is a matrix formed using components of the outward normal vector on the boundary $\Gamma^{(k)}$. For $2 \mathrm{D}$ problems, it is written as:

$$
n^{(k)}(x)=\left[\begin{array}{cc}
n_{x}^{(k)} & 0 \\
0 & n_{y}^{(k)} \\
n_{y}^{(k)} & n_{x}^{(k)}
\end{array}\right] .
$$

Usually, the trial function $u^{h}(x)$ for SFEM is the same as that for the FEM (Eq. (11)) and therefore, the force vector $f$ in SFEM is written similar to that in FEM.

Substituting Eq. (11) into Eq. (20), the smoothed strain on the smoothing domain $\Omega^{(k)}$ can be written based on nodal displacements:

$$
\tilde{\varepsilon}_{k}=\sum_{I \in N^{(k)}} \tilde{B}_{I}\left(x_{k}\right) d_{I}
$$

where $N^{(k)}$ is the number of nodes that are directly connected to node $k$ for NSFEM and total number of nodes of elements containing the common edge $i$ for ESFEM. For CSFEM, $N^{(k)}$ is the number of nodes of the element that contains the current cell. $\tilde{B}_{I}\left(x_{k}\right)$ is termed the smoothed strain matrix on the smoothing domain $\Omega^{(k)}$ :

$$
\tilde{B}_{I}\left(x_{k}\right)=\left[\begin{array}{cc}
\tilde{b}_{I x}\left(x_{k}\right) & 0 \\
0 & \tilde{b}_{I y}\left(x_{k}\right) \\
\tilde{b}_{I y}\left(x_{k}\right) & \tilde{b}_{I x}\left(x_{k}\right)
\end{array}\right],
$$

and is calculated using this equation: 


$$
\tilde{b}_{I h}\left(x_{k}\right)=\frac{1}{A^{(k)}} \int_{\Gamma^{(k)}} n_{h}^{(k)}(x) N_{I}(x) d \Gamma(h=x, y) .
$$

A linear compatible displacement field along the boundary $\Gamma^{(k)}$ needs only one Gaussian point for line integration along each segment of boundary $\Gamma_{i}^{(k)}$ of $\Omega^{(k)}$. In that case, the above equation can be further simplified as:

$$
\tilde{b}_{I h}\left(x_{k}\right)=\frac{1}{A^{(k)}} \sum_{i=1}^{M} n_{i h}^{(k)} N_{I}\left(x_{i}^{G P}\right) l_{i}^{(k)}(h=x, y),
$$

where $M$ is the total number of boundary segments of $\Gamma_{i}^{(k)}$ and $x_{i}^{G P}$ is the midpoint (Gaussian point) of the boundary segment of $\Gamma_{i}^{(k)}$, with its length and outward unit normal denoted by $l_{i}^{(k)}$ and $n_{i h}^{(k)}$, respectively.

Eq. (25) shows that in SFEM, only shape function values at some particular points along segments of boundary $\Gamma_{i}^{(k)}$ are needed and no explicit analytical form is required.

Using triangular elements, the smoothed strain matrix $\tilde{B}_{I}\left(x_{k}\right)$ can be written in another way:

$$
\tilde{B}_{I}\left(x_{k}\right)=\frac{1}{A^{(k)}} \sum_{j=1}^{N_{e}^{(k)}} \frac{1}{3} A_{e}^{(j)} B_{j},
$$

where $N_{e}^{(k)}$ is the number of elements associated with the smoothing domain $k ; A_{e}^{(j)}$ and $B_{j}$ are the area and the strain matrix of the $j$ th element associated with the smoothing domain $k$, respectively; and $A^{(k)}$ is calculated as:

$$
A^{(k)}=\int_{\Omega^{(k)}} d \Omega=\frac{1}{3} \sum_{j=1}^{N_{e}^{(k)}} A_{e}^{(j)} .
$$

As can be seen in this formulation, only the area and the usual compatible strain matrices, $B_{j}$, (Eq. (16)) of triangular elements are needed to calculate the global stiffness matrix for SFEM.

The global stiffness matrix $\tilde{K}$ is then assembled by a procedure similar to the FEM:

$$
\tilde{K}_{I J}=\sum_{k=1}^{N_{n}} \tilde{K}_{I J}^{(k)}
$$

where $N_{n}$ is the number of smoothing domains and $\tilde{K}_{I J}^{(k)}$ is the smoothed stiffness matrix calculated on the smoothing domain $\Omega^{(k)}$ that is calculated by:

$$
\tilde{K}_{I J}^{(k)}=\int_{\Omega^{(k)}} \tilde{B}_{I}^{T} D \tilde{B}_{J} d \Omega=\tilde{B}_{I}^{T} D \tilde{B}_{J} A^{(k)} .
$$

\section{Numerical formulation}

Spatial discretization of Biot's consolidation equations is done based on smoothed finite element method, as described in the previous section. After discretization, the first and second partial differential equations for coupled analysis of porous media (Eqs. (8) and (9)) will become:

$$
\begin{aligned}
& {\left[\tilde{\mathbf{k}}_{m}\right]\{\mathbf{u}\}+[\tilde{\mathbf{c}}]\left\{\mathbf{u}_{w}\right\}=\{\mathbf{f}\},} \\
& {\left[\tilde{\mathbf{c}}^{T}\left\{\frac{d \mathbf{u}}{d t}\right\}-\left[\tilde{\mathbf{k}}_{c}\right]\left\{\mathbf{u}_{w}\right\}=\{0\},\right.}
\end{aligned}
$$

where $\left[\tilde{\mathbf{k}}_{m}\right]$ is the smoothed mechanical stiffness, $\left[\tilde{\mathbf{k}}_{c}\right]$ is the smoothed fluid conductivity, and $[\tilde{\mathbf{c}}]$ is the smoothed coupling matrix. Furthermore, $\{\mathbf{f}\}$ is the external loading and body forces, $\{\mathbf{u}\}$ is nodal displacements, and $\left\{\mathbf{u}_{w}\right\}$ is nodal excess pore pressures vector.

As described in the previous section, the smoothed mechanical stiffness matrix $\tilde{\mathbf{k}}_{m}$ and the smoothed fluid conductivity matrix $\tilde{\mathbf{k}}_{c}$ are written as:

$$
\begin{gathered}
\tilde{\mathbf{k}}_{m}=\sum_{k=1}^{N_{S D}} \tilde{\mathbf{k}}_{m}^{(k)}=\sum_{k=1}^{N_{S D}} \tilde{\mathbf{B}}^{T} \mathbf{D} \tilde{\mathbf{B}} A^{k}, \\
\tilde{\mathbf{k}}_{c}=\sum_{k=1}^{N_{S D}} \tilde{\mathbf{k}}_{c}^{(k)}=\sum_{k=1}^{N_{S D}} \tilde{\mathbf{T}}^{T} \mathbf{K} \tilde{\mathbf{T}} A^{k} .
\end{gathered}
$$

Here, $N_{S D}$ is the number of smoothing domains and $A^{k}$ is the area of each one. These equations are basically the same, where $\tilde{\mathbf{B}}$ and $\tilde{\mathbf{T}}$ are similar and represent the mechanical and hydraulic smoothed gradient matrices, as described for $\tilde{\mathbf{B}}$ previously. It is also restated that $\mathbf{D}$ and $\mathbf{K}$ are matrices of elastic moduli and permeability, respectively. Summation here indicates the assembly procedure to form the global matrices from smoothing domain matrices, similar to the conventional FEM in which global matrices are formed using the element matrices.

Another main matrix in Eqs. (30) and (31) is the smoothed coupling matrix, $\tilde{\mathbf{c}}$. In two dimensions, generally, the coupling matrix is formed by the following integration:

$$
\iint \frac{\partial N_{u}}{\partial x} N_{p} d x d y
$$

The first derivative here comes from displacement shape functions, and the second term indicates pore pressure shape functions. Using SFEM, the smoothed coupling matrix is written as:

$$
\tilde{\mathbf{c}}=\sum_{k=1}^{N_{S D}} \tilde{\mathbf{c}}^{(k)}=\sum_{k=1}^{N_{S D}}\left(\int_{\Omega^{(k)}} \tilde{\mathbf{B}}_{c} \mathbf{N}_{p} d \Omega\right) .
$$

Using Eq. (24), $\tilde{\mathbf{B}}_{c}$ is defined as:

$$
\tilde{\mathbf{B}}_{c}=\left[\begin{array}{ll}
\tilde{b}_{I x}\left(x_{k}\right) & \tilde{b}_{I y}\left(x_{k}\right)
\end{array}\right],
$$

and $\mathbf{N}_{p}$ is matrix of pore pressure shape functions.

In Eqs. (32) and (33), since gradient matrices ( $\tilde{\mathbf{B}}$ 
and $\tilde{\mathbf{T}})$ are constant over each smoothing domain, no transformation to natural coordinates is needed, and $\tilde{\mathbf{k}}_{m}$ and $\tilde{\mathbf{k}}_{c}$ are easily evaluated. However, calculation of $\tilde{\mathbf{c}}$ is not readily done, because $\mathbf{N}_{p}$ is not constant over each smoothing domain. Here, $\tilde{\mathbf{B}}_{c}$ is constant over each smoothing domain, so we will have:

$$
\tilde{\mathbf{c}}=\sum_{k=1}^{N_{S D}} \tilde{\mathbf{B}}_{c}\left(\int_{\Omega^{(k)}} \mathbf{N}_{p} d \Omega\right) .
$$

The integration in this equation is evaluated over each smoothing domain, using the Gauss integration method with proper number of quadrature points.

Writing Eq. (30) in incremental form, the first equation of coupled system will be obtained; then, by linear interpolation in time using the $\theta$ method, we will have:

$$
\{\Delta \mathbf{u}\}=\Delta t\left((1-\theta)\left\{\frac{d \mathbf{u}}{d t}\right\}_{0}+\theta\left\{\frac{d \mathbf{u}}{d t}\right\}_{1}\right) .
$$

Now, Eq. (31) can be written based on $\theta$ method to give expressions for derivatives. After some simplifications, this final global system of equations will be achieved for coupled analysis of porous media using Biot's equations and SFEM:

$$
\left[\begin{array}{cc}
{\left[\tilde{\mathbf{k}}_{m}\right]} & {[\tilde{\mathbf{c}}]} \\
{[\tilde{\mathbf{c}}]^{T}} & -\theta \Delta t\left[\tilde{\mathbf{k}}_{c}\right]
\end{array}\right]\left\{\begin{array}{c}
\{\Delta \mathbf{u}\} \\
\left\{\Delta \mathbf{u}_{w}\right\}
\end{array}\right\}=\left\{\begin{array}{c}
\{\Delta \mathbf{f}\} \\
\Delta t\left[\tilde{\mathbf{k}}_{c}\right]\left\{\mathbf{u}_{w}\right\}_{0}
\end{array}\right\} .
$$

$\theta$ can vary from zero (fully explicit scheme) to 1.0 (fully implicit scheme). The approximation is unconditionally stable when $\theta \geq 0.5$, but for any value of $\theta \neq 1$, the numerical solution can exhibit a spurious ripple effect [1].

\section{Numerical examples}

In coupled hydro-mechanical problems, when permeability approaches zero and/or solid and fluid phases become incompressible, there may be a spurious oscillation in results for pressure field. It occurs when the same order shape functions (without any stabilization technique) are used for both mechanical and hydraulic components. To prevent this problem, elements have to satisfy the so-called Babuška-Brezzi condition $[29,30]$ or the patch test [31]. These conditions basically state that the shape function used for interpolation of mechanical field must be of an order higher than the shape function used for interpolation of hydraulic field.

In the conventional FEM, to satisfy the BabuškaBrezzi condition or the patch test, mixed elements with different orders of shape functions are used. For example, 6-node triangles (T6) for mechanical field are combined with 3-node triangles (T3) for hydraulic field. Prevention of spurious oscillatory results in SFEM is more complicated and needs more considerations.
Many methods for creation of a coupled SFEM with acceptable results have been investigated; two of them, which have shown better performance, are discussed here.

\subsection{Different coupled hydro-mechanical SFEM models}

A major shortcoming of smoothed finite element methods is that using higher order elements deteriorates their accuracy. SFEMs using quadratic 8-node quadrilateral elements (Q8) does not pass the patch test [32]. Further investigation by authors showed the same shortcoming for quadratic 6-node triangular elements. When strain smoothing is applied to higher order elements, nonlinear gradients are replaced with piecewise-linear smoothed gradients, which reduces the accuracy of solution. As a result, mixed elements are not applicable for the solution to the coupled hydromechanical problems using SFEMs.

Since the mixed elements method is not applicable in SFEM, a number of other methods were investigated to create stable coupled models. The first method discussed here is based on different properties of different types of SFEM. A major cause of oscillatory results in coupled analysis is volumetric locking of hydraulic phase, because stiffness of this phase is very much higher than that of the solid phase [31]. On the other hand, ESFEM gives stiffer results than the exact solution does, while NSFEM is volumetric locking free [8]. In this way, ESFEM may be used for the mechanical part to give ultra-accurate results, and NSFEM for hydraulic part to solve the volumetric locking problem, which causes spurious oscillations. The model created by this method is a mixed ESFEM/NSFEM model.

When mixed T6/T3 elements are used in the conventional FEM, T3 elements reduce overall accuracy and may cause problems such as volumetric locking, due to their low order. Another method for creation of coupled SFEM models is intended to overcome this problem. Application of strain smoothing only to T3 elements (and leaving alone T6 elements) improves their performance and makes them more accurate [8]. Using this method, only the fluid conductivity matrix $\mathbf{k}_{c}$ in Eq. (39) is smoothed and other components of the overall stiffness matrix are written as in the conventional FEM.

Performance of these different strategies for application of SFEMs in the solution to coupled hydromechanical problems is investigated through the solution to typical consolidation problems. Results are obtained using a developed SFEM/FEM code. SFEM results are compared with the FEM and analytical solutions.

\subsection{One-dimensional consolidation analysis}

The problem consists of a saturated layer of soil, with 


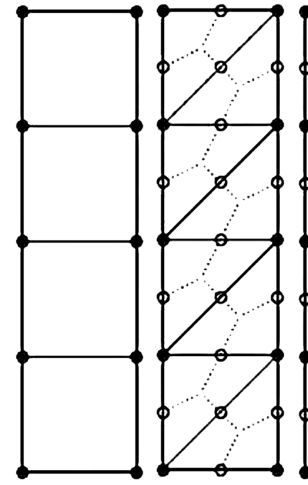

(a)

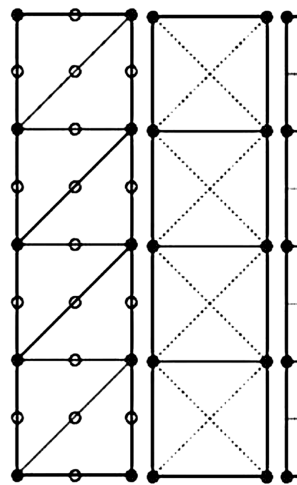

(c)

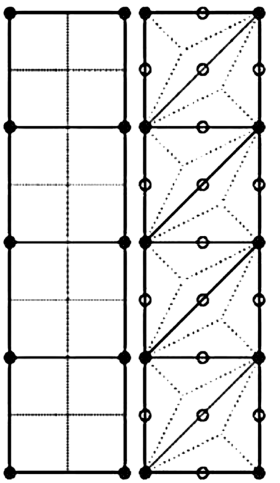

(e) (f)

Figure 4. Discretization of problem domain in different ways (o: nodes for mechanical part, *: nodes for hydraulic part): (a) FEMQ4(mech.)/FEMQ4(hyd.), (b)

FEMT6(mech.)/NSFEMT3(hyd.), (c)

FEMT6(mech.)/FEMT3(hyd.), (d) ESFEMQ4, (e)

NSFEMQ4, and (f) FEMT6(mech.)/ESFEMT3(hyd.).

thickness $H=10 \mathrm{~m}$ and large horizontal extent, considered to be one-dimensional. Standard geotechnical boundary conditions are imposed for displacements and soil layer is only drained on top. Problem domain, discretized in different ways, is depicted in Figure 4. When quadrilateral elements are used, problem domain is discretized into 4 elements with sides' length of $2.5 \mathrm{~m}$, and when triangular elements are used, problem domain is discretized into 8 elements with perpendicular sides of the same length. Other parameters are: $\theta=1$, elasticity modulus $E=10000 \mathrm{kPa}$, Poisson's ratio $\nu=0$, and permeability in vertical direction $K_{y}=5 \times 10^{-8} \mathrm{~m} / \mathrm{s}$ and in horizontal direction $K_{x}=0$, since the problem is considered to be one-dimensional. A surcharge of $\sigma=20 \mathrm{kPa}$ is applied on the surface and body loads are ignored. Analyses are performed in 700 time steps with a uniform size of $8640 \mathrm{~s}$ (0.1 day), which represent a total of 70 days.

The closed form solution to one-dimensional consolidation is given by Terzaghi et al. [33] as follows:

Excess pore water pressure:

$$
P=\frac{4}{\pi} \sigma \sum_{n=1}^{\infty} \frac{1}{2 n-1} \sin \left(\frac{(2 n-1) \pi y}{2 H}\right) e^{-(2 n-1)^{2} \frac{\pi^{2}}{4} T_{v}}
$$

Degree of consolidation:

$$
U_{t}=1-\frac{8}{\pi^{2}} \sum_{n=1}^{\infty} \frac{1}{(2 n-1)^{2}} e^{-(2 n-1)^{2} \frac{\pi^{2}}{4} T_{v}} .
$$

Surface settlement:

$$
S_{t}=U_{t} m_{v} \sigma H
$$

where:

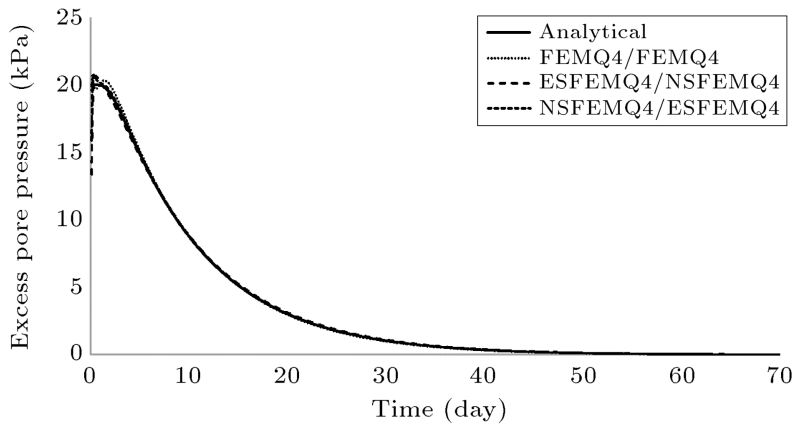

(a)

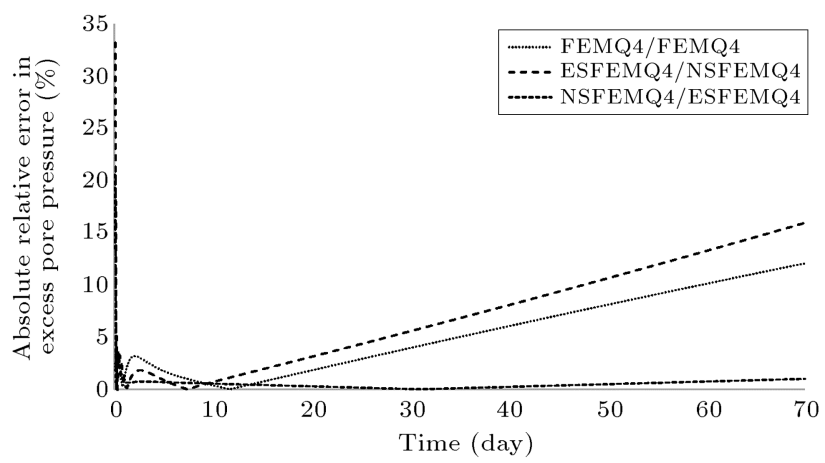

(b)

Figure 5. (a) Excess pore pressure at the bottom of domain for one-dimensional consolidation problem using different methods. (b) Absolute relative error in excess pore pressure results (the first method).

$$
\begin{aligned}
& T_{v}=\frac{C_{v}}{H^{2}} t, \\
& C_{v}=\frac{k}{\gamma_{w} m_{v}}, \\
& m_{v}=\frac{(1+\nu)(1-2 \nu)}{E(1-\nu)} .
\end{aligned}
$$

First, performance of the mixed ESFEM/NSFEM model is investigated. Initial analyses show that application of T3 elements in this method will result in large errors; therefore, the related analyses are not mentioned. However, the results of Q4 elements are notable and discussed here. Although it seems more rational to use ESFEM for mechanical and NSFEM for hydraulic parts, as discussed previously, each type of SFEM is used for both hydraulic and mechanical parts to make a thorough investigation.

Excess pore pressure results, obtained at the bottom of the domain, are shown in Figure 5 . In Figure 5(a), analytical results are shown and FEMQ4(mech.)/FEMQ4(hyd.) results are compared with ESFEMQ4(mech.)/NSFEMQ4(hyd.) and NSFEMQ4(mech.)/ESFEMQ4(hyd.) results. As discussed before, FEMQ4/FEMQ4 model does not satisfy the Babuška-Brezzi condition, and initial oscillations are obvious in the results of the first time steps. 
However, possibility of using different strain smoothing technics instead of mixed elements method is open to question in the research; therefore, this model is analyzed for comparison purposes. Furthermore, effect of using different strain smoothing technics for mechanical and hydraulic parts is investigated.

Figure 5(b) shows the absolute relative error in results, compared with analytical ones. It is shown that, contrary to what was expected, NSFEMQ4/ESFEMQ4 approach reduces overall errors of the FEM with the same order elements much more efficiently than ESFEMQ4/NSFEMQ4, and the latter approach even increases FEM errors in most time steps. Although NSFEMQ4/ESFEMQ4 method greatly reduces errors of the FEM using the same order elements, initial oscillations are not still acceptable. As a result, it is concluded that using different smoothing technics cannot replace using mixed elements.

After revealing shortcomings of the first method, the second one is investigated. As described before, the other method is to apply SFEM only for hydraulic part of the mixed T6/T3 elements, which uses lower order T3 elements. All three smoothing technics are possible to use with this method. Among them, cell based method gives identical results with FEM when using T3 elements [8] and is not discussed here. Results are compared with FEM T6(mech.)/T3(hyd.) and analytical ones. Figure 6 shows excess pore pressure results, obtained at the bottom of the domain.

In Figure 6(a), results of the method using two different smoothing technics are compared. It is shown that while using NSFEM for hydraulic part of T6/T3 elements deteriorates the results, ESFEM makes them closer to analytical ones. As concluded in investigation of the first method, node based technic is not suitable for smoothing the hydraulic part in coupled hydromechanical formulations. This is possibly because of its over-softness that makes it unstable in temporal analyses. However, while NSFEM increases the error in excess pore pressure results, ESFEM can make them more accurate.

Figure 6(b) shows absolute relative errors. Here, it is shown that when using an identical mesh Figure 4, replacing FEMT3 with ESFEMT3 in T6/T3 elements greatly improves the accuracy. On a same computer, analysis using FEMT6/ESFEMT3 elements lasted less than twice the computational time needed for FEMT6/FEMT3 elements. On the other hand, taking an average for all time steps in Figure 6(b) shows that using FEMT6/ESFEMT3 has reduced the mean relative error by nearly four times.

The conventional method for increasing the accuracy of T3 elements in FEMT6/FEMT3 models increases the order of the shape function of T3 elements and uses T6 elements instead. However, when T6 elements are also used for the mechanical part, this

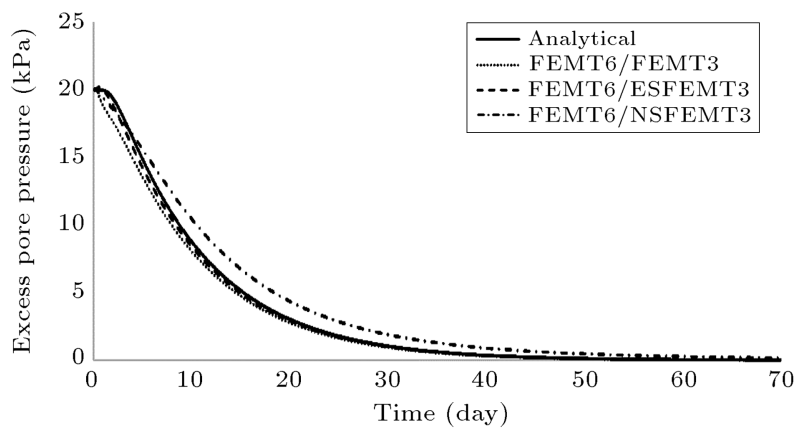

(a)

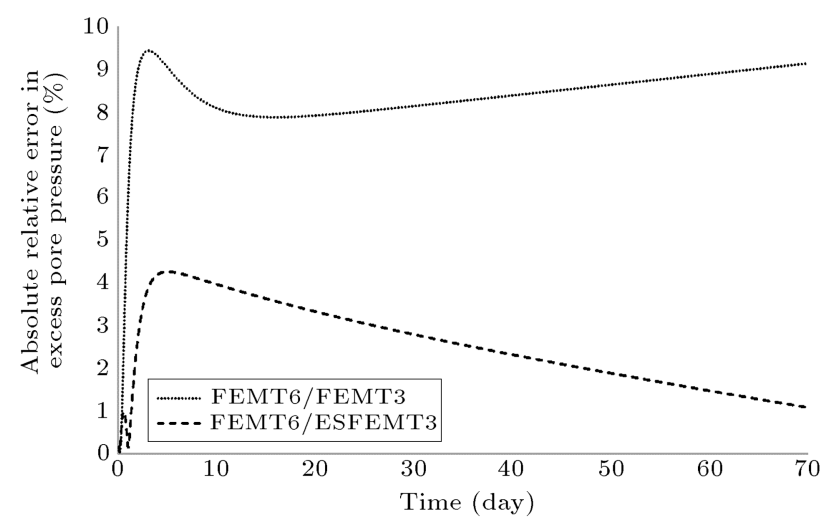

(b)

Figure 6. (a) Excess pore pressure at the bottom of domain for one-dimensional consolidation problem using different methods. (b) Absolute relative error in excess pore pressure results (the second method).

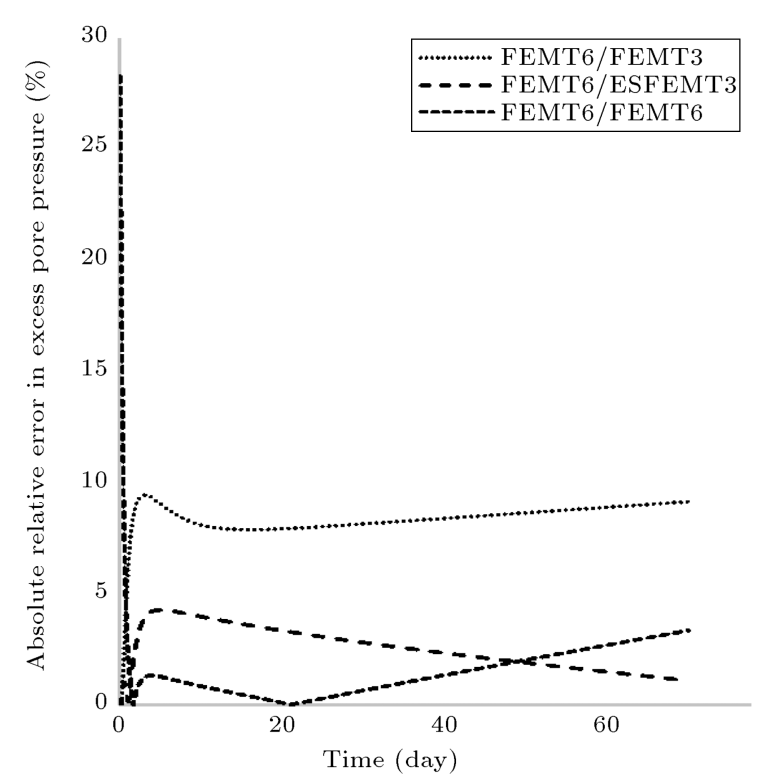

Figure 7. Absolute relative error in excess pore pressure results using different methods.

is not feasible. As discussed before, FEMT6/FEMT6 model violates the Babuška-Brezzi condition. Figure 7 shows spurious oscillation in the initial results of this model, although it can greatly decrease the overall 


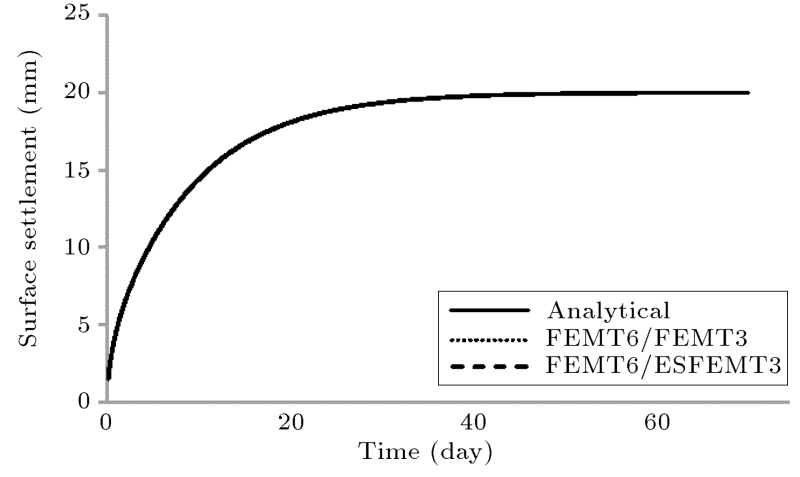

(a)

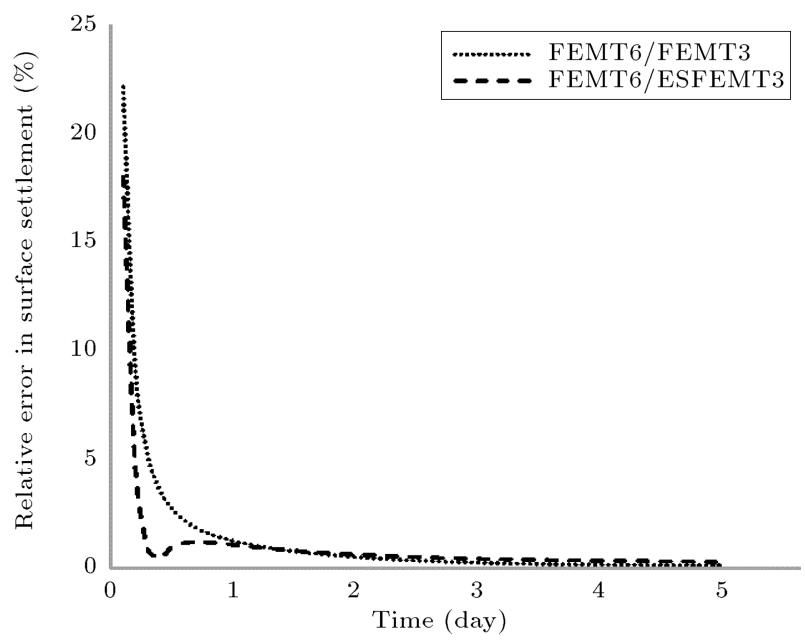

(b)

Figure 8. (a) Surface settlement for one-dimensional consolidation problem using different methods. (b) Relative error in surface settlement.

absolute relative error in excess pore pressure. The remaining conventional option for increasing the accuracy of FEMT6/FEMT3 models is replacing both T6 and T3 elements with higher order elements. But, Figure 7 shows that using FEMT6/ESFEMT3 is an appropriate method to improve the accuracy of FEMT6/FEMT3 elements, without using higher order elements that need high computational effort. Error reduction of this method is comparable to using high order elements.

Figure 8 shows surface settlement results for this problem. In Figure 8(a), results using different methods seem identical to analytical ones. However, a closer look on relative error in the first five days in Figure 8(b) reveals that FEMT6/ESFEMT3 gives results with less error for settlement than FEMT6/FEMT3 does. It is concluded that for the current problem, strain smoothing in FEMT6/ESFEMT3 can give more accurate results for both excess pore pressure and settlement.

To study the effect of mesh properties on performance of the second method, the problem described in this section was solved with different meshes. Problem

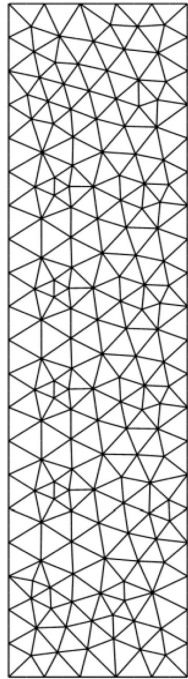

$H_{\max }=0.5$

Elements $=334$

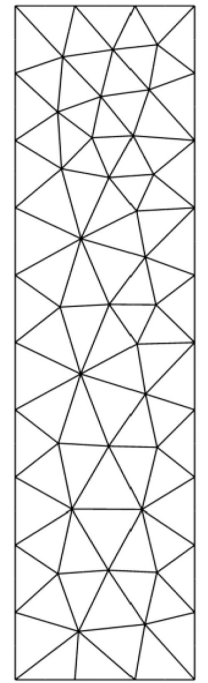

$H_{\max }=1.0$

Elements $=84$

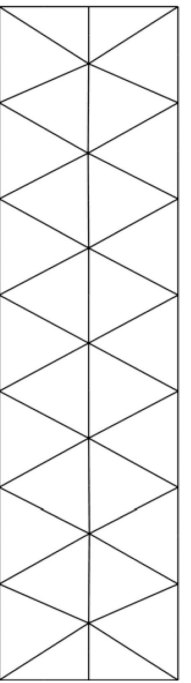

$H_{\max }=1.5$

Elements $=30$

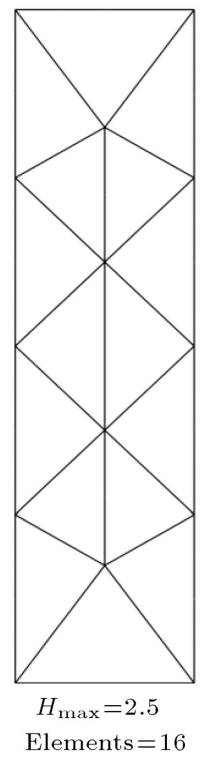

Figure 9. Different types of domain discretization.

domain was discretized using 4 different unstructured triangular meshes with maximum edge lengths $\left(H_{\max }\right)$ of $2.5,1.5,1$, and $0.5 \mathrm{~m}$. Meshes are depicted in Figure 9 and number of elements for each one is indicated. Both FEMT6/FEMT3 and FEMT6/ESFEMT3 models were solved with identical discretization and results for excess pore pressure are compared. Figure 10 shows absolute relative error in excess pore pressure results of both methods for different meshes. In all cases, although error reduction is not observed for every time step, the FEMT6/ESFEMT3 elements have less error in the initial ones. Since the results do not have an obvious trend, a general procedure to choose element and time step sizes for creation of efficient coupled SFEM models, using the proposed method, is recommended in future research.

\section{Conclusion}

In this paper, considering the Biot's consolidation theory, smoothed finite element methods were introduced and numerical formulation of coupled hydromechanical problems using SFEM was described. Two methods for creation of coupled SFEM models were advised: one by use of mixed ESFEM/NSFEM elements, and the other by smoothing only the lower order elements of the hydraulic part in FEMT6/FEMT3 models. It was shown that although using mixed ESFEM/NSFEM method could improve accuracy of the results of the FEM model using same order Q4 elements, large initial oscillations in excess pore pressure results would make the method not applicable. It was also concluded that while using ESFEM for hydraulic part reduced overall errors, switching the method by NSFEM deteriorated the results, contrary 

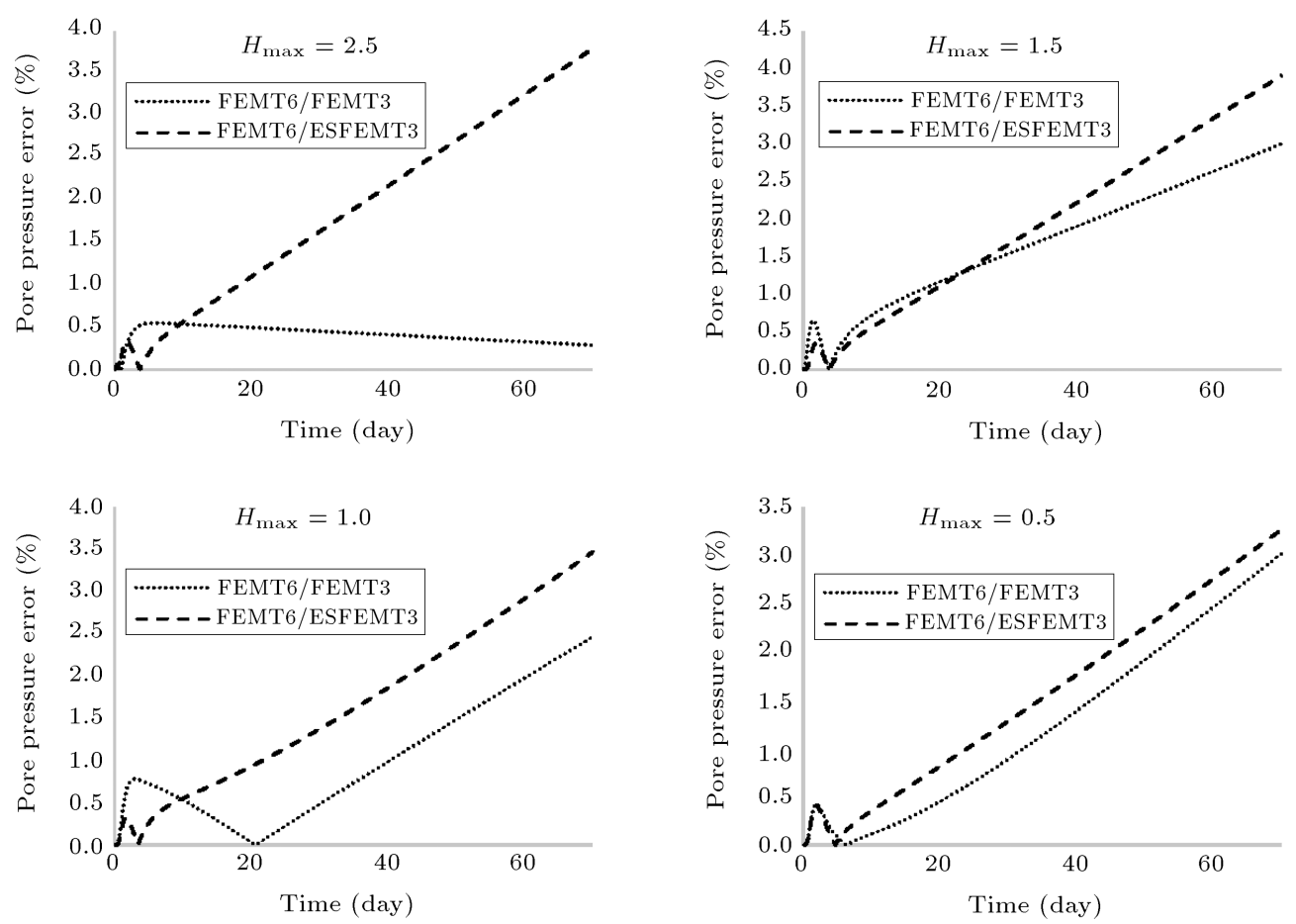

Figure 10. Absolute relative error in excess pore pressure results of analyses using different meshes.

to what was expected. Further investigation revealed the same condition for performance of the node based and edge based methods in smoothing the hydraulic part of mixed FEMT6/FEMT3 elements. In the second method, by using strain smoothing for T3 elements to make them FEMT6/ESFEMT3, performance was improved and both excess pore pressure and settlement results became more accurate. It was shown that using FEMT6/ESFEMT3 elements was an appropriate method to improve the accuracy of FEMT6/FEMT3 models, without using higher order elements. However, selection of appropriate element and time step sizes needs further research.

\section{References}

1. Oliaei, M.N. and Pak, A. "Element free Galerkin meshless method for fully coupled analysis of consolidation process", Scientia Iranica, Transaction A, 16(1), pp. 65-77 (2009).

2. Oliaei, M.N., Soga, K., and Pak, A. "Some numerical issues using element-free Galerkin mesh-less method for coupled hydro-mechanical problems", International Journal for Numerical and Analytical Methods in Geomechanics, 33(7), pp. 915-938 (2009).

3. Oliaei, M.N., Pak, A., and Soga, K. "A coupled hydromechanical analysis for prediction of hydraulic fracture propagation in saturated porous media using EFG mesh-less method", Computers and Geotechnics, 55, pp. 254-266 (2014).

4. Khoshghalb, A. and Khalili, N. "A stable meshfree method for fully coupled flow-deformation analysis of saturated porous media", Computers and Geotechnics, 37(6), pp. 789-795 (2010).

5. Beissel, S. and Belytschko, T. "Nodal integration of the element-free Galerkin method", Computer Methods in Applied Mechanics and Engineering, 139(1), pp. 49-74 (1996).

6. Chen, J.S., Wu, C.T., Yoon, S., and You, Y. "A stabilized conforming nodal integration for Galerkin meshfree methods", International Journal for Numerical Methods in Engineering, 50(2), pp. 435-466 (2001).

7. Liu, G.R., Li, Y., Dai, K.Y., Luan, M.T., and Xue, W. "A linearly conforming radial point interpolation method for solid mechanics problems", International Journal of Computational Methods, 3(04), pp. 401-428 (2006).

8. Liu, G. and Nguyen, T., Smoothed Finite Element Methods, 1st Edn., CRC Press, Boca Raton, USA (2010).

9. Liu, G.R., Dai, K.Y., and Nguyen, T.T. "A smoothed finite element method for mechanics problems", Computational Mechanics, 39(6), pp. 859-877 (2007).

10. Liu, G.R., Nguyen-Thoi, T., Nguyen-Xuan, H., and Lam, K.Y. "A node-based smoothed finite element method (NS-FEM) for upper bound solutions to solid mechanics problems", Computers \& Structures, 87(1), pp. 14-26 (2009).

11. Liu, G.R., Nguyen-Thoi, T., and Lam, K.Y. "An edgebased smoothed finite element method (ES-FEM) for static, free and forced vibration analyses of solids", Journal of Sound and Vibration, 320(4), pp. 1100-1130 (2009). 
12. Nguyen-Thoi, T., Phung-van, P., Rabczuk, T., Nguyen-Xuan, H., and Le-Van, C. "Free and forced vibration analysis using the n-sided polygonal cell-based smoothed finite element method (nCS-FEM)", International Journal of Computational Methods, 10(01), p. 1340008 (2013).

13. Cui, X.Y., Hu, X.B., Li, G.Y., and Liu, G.R. "A modified smoothed finite element method for static and free vibration analysis of solid mechanics", International Journal of Computational Methods, 13(06), p. 1650043 (2016).

14. Cui, X.Y., Liu, G.R., Li, G.Y., Zhang, G.Y., and Sun, G.Y. "Analysis of elastic-plastic problems using edgebased smoothed finite element method", International Journal of Pressure Vessels and Piping, 86(10), pp. 711-718 (2009).

15. Lee, K., Son, Y., and Im, S. "Three-dimensional variable-node elements based upon CS-FEM for elastic-plastic analysis", Computers \& Structures, 158, pp. 308-332 (2015).

16. Liu, G.R., Chen, L., Nguyen-Thoi, T., Zeng, K.Y., and Zhang, G.Y. "A novel singular node-based smoothed finite element method (NS-FEM) for upper bound solutions of fracture problems", International Journal for Numerical Methods in Engineering, 83(11), pp. 1466-1497 (2010).

17. Zeng, W., Liu, G.R., Kitamura, Y., and NguyenXuan, H. "A three-dimensional ES-FEM for fracture mechanics problems in elastic solids", Engineering Fracture Mechanics, 114, pp. 127-150 (2013).

18. Liu, G.R., Chen, L., and Li, M. "S-FEM for fracture problems, theory, formulation and application", International Journal of Computational Methods, 11(03), p. 1343003 (2014).

19. Li, E., Zhang, Z., He, Z.C., Xu, X., Liu, G.R., and Li, Q. "Smoothed finite element method with exact solutions in heat transfer problems", International Journal of Heat and Mass Transfer, 78, pp. 1219-1231 (2014).

20. Cui, X.Y., Li, Z.C., Feng, H., and Feng, S.Z. "Steady and transient heat transfer analysis using a stable node-based smoothed finite element method", International Journal of Thermal Sciences, 110, pp. 12-25 (2016).

21. He, Z.C., Li, G.Y., Liu, G.R., Cheng, A.G., and Li, E. "Numerical investigation of ES-FEM with various mass re-distribution for acoustic problems", Applied Acoustics, 89, pp.222-233 (2015).

22. Wang, G., Cui, X.Y., Feng, H., and Li, G.Y. "A stable node-based smoothed finite element method for acoustic problems", Computer Methods in Applied Mechanics and Engineering, 297, pp. 348-370 (2015).

23. Chai, Y.B., Li, W., Gong, Z.X., and Li, T.Y. "Hybrid smoothed finite element method for two dimensional acoustic radiation problems", Applied Acoustics, 103, pp. 90-101 (2016).
24. Li, Y., Zhang, G.Y., Liu, G.R., Huang, Y.N., and Zong, Z. "A contact analysis approach based on linear complementarity formulation using smoothed finite element methods", Engineering Analysis with Boundary Elements, 37(10), pp. 1244-1258 (2013).

25. Nguyen-Xuan, H., Liu, G.R., Bordas, S., Natarajan, S., and Rabczuk, T. "An adaptive singular ES-FEM for mechanics problems with singular field of arbitrary order", Computer Methods in Applied Mechanics and Engineering, 253, pp. 252-273 (2013).

26. Nguyen-Xuan, H., Wu, C.T., and Liu, G.R. "An adaptive selective ES-FEM for plastic collapse analysis", European Journal of Mechanics-A/Solids, 58, pp. 278290 (2016).

27. Kumar, V. and Metha, R. "Impact simulations using smoothed finite element method", International Journal of Computational Methods, 10(04), p. 1350012 (2013).

28. Biot, M.A. "General theory of three-dimensional consolidation", Journal of Applied Physics, 12(2), pp. 155164 (1941).

29. Babuška, I. "The finite element method with Lagrange multipliers", Numerische Mathematik, 20(3), pp. 179192 (1973).

30. Brezzi, F. "On the existence, uniqueness and approximation of saddle-point problems arising from Lagrangian multipliers", Revue Française Dáutomatique, Informatique, Recherche Opé Rationnelle. Analyse Numérique, 8(2), pp. 129-151 (1974).

31. Zhu, J.Z., Taylor, Z.R.L., and Zienkiewicz, O.C., The Finite Element Method: Its Basis and Fundamentals, 7th Edn., Elsevier, USA (2013).

32. Bordas, S., Natarajan, S., Kerfriden, P., Augarde, C.E., Mahapatra, D.R., Rabczuk, T., and Pont, S.D. "On the performance of strain smoothing for quadratic and enriched finite element approximations (XFEM/GFEM/PUFEM)", International Journal for Numerical Methods in Engineering, 86(4-5), pp. 637666 (2011).

33. Terzaghi, K., Peck, R.B., and Mesri, G., Soil Mechanics in Engineering Practice, 3rd Edn., John Wiley \& Sons, USA (1996).

\section{Biographies}

Erfan Karimian is a $\mathrm{PhD}$ candidate in Civil Engineering (Geotechnical Engineering) at Tarbiat Modares University, Tehran, Iran. He graduated with MSc in Civil Engineering (Geotechnical Engineering) from Shiraz University in 2011. He specializes in applications of numerical methods in geotechnical engineering.

Mohammad Oliaei is Assistant Professor of Civil Engineering. He joined the Geotechnical Group in the Department of Civil Engineering, Tarbiat Modares 
University, in 2008. He graduated with $\mathrm{PhD}$ from Sharif University of Technology in 2007 as the first rank student. In 2005, he was awarded a scholarship from British Council to continue his $\mathrm{PhD}$ studies at
Cambridge University. He specializes in the area of geotechnical engineering and numerical modelling (especially meshless and DEM). He is the reviewer of some ISI and Scientific and Technical Journals. 